\title{
A new theory for perpendicular transport of cosmic rays
}

\author{
A. Shalchi and I. Kourakis \\ Institut für Theoretische Physik, Lehrstuhl IV: Weltraum- und Astrophysik, Ruhr-Universität Bochum, 44780 Bochum, Germany \\ e-mail: andreasm4@yahoo.com
}

Received 8 February 2007 / Accepted 21 April 2007

\section{ABSTRACT}

\begin{abstract}
We present an improved nonlinear theory for the perpendicular transport of charged particles. This approach is based on an improved nonlinear treatment of field-line random walk in combination with a generalized compound diffusion model. The generalized compound diffusion model employed is more systematic and reliable, in comparison with previous theories. Furthermore, the theory shows remarkably good agreement with test-particle simulations and solar wind observations.
\end{abstract}

Key words. diffusion - scattering - turbulence - gamma rays: theory

\section{Introduction}

The long-standing problem of particle transport perpendicular to a magnetic background field in turbulent magnetized plasmas is revisited in this article. Although this problem has been discussed in several papers (e.g. Jokipii 1966; Bieber \& Matthaeus 1997; Kóta \& Jokipii 2000; Matthaeus et al. 2003; Shalchi et al. 2004; Webb et al. 2006; Shalchi 2006), a final solution has not been provided so far. The perpendicular transport of charged particles is a central problem in astrophysics, since the knowledge of the diffusion tensor for particle transport parallel and perpendicular to the prescribed external magnetic field is essential for describing solar energetic particles (Dröge 2000), the modulation of Galactic cosmic rays (Burger \& Hattingh 1998), diffusive shock acceleration, and the lifetime of cosmic rays in the Galaxy (Jokipii \& Parker 1969). Furthermore, there are measurements of perpendicular mean free paths in the heliosphere (Chenette et al. 1977; Palmer 1982; Burger et al. 2000) that still await a theoretical explanation.

An early treatment of particle transport has relied on a quasilinear description of cosmic ray propagation (Jokipii 1966). In the quasilinear theory (QLT) it is assumed that particles follow the magnetic field-lines while they move unperturbed in the direction parallel to the background field. Thus, the corresponding result is often referred to as the field-line random walk limit (FLRW-limit). According to this result, which has originally been derived for the slab turbulence model, the perpendicular mean-square deviation (MSD) of the particle increases linearly with time, viz. $\left\langle(\Delta x)^{2}\right\rangle=2 \kappa_{x x} t$. This linear time dependence is usually referred to as a classical Markovian diffusion process. Thirty-four years later, Kóta \& Jokipii (2000) formulated a compound diffusion model that assumes that the particle moves along the magnetic field-lines while it is scattered diffusively in the parallel direction. Relying on the Taylor-Green-Kubo-formulation (e.g. Kubo 1957), in combination with the assumption of diffusive field-line wandering, Kóta $\&$ Jokipii (2000) have found a subdiffusive behavior of particle transport of the form $\left\langle(\Delta x)^{2}\right\rangle \sim \sqrt{t}$. In the same years, particle propagation in magnetized plasmas was explored by making use of test-particle simulations (e.g. Giacalone \& Jokipii 1999;
Mace et al. 2000; Qin et al. 2002a,b), where it was clearly confirmed that $\left\langle(\Delta x)^{2}\right\rangle \sim \sqrt{t}$, so long as a slab model is considered for the turbulence geometry (see Qin et al. 2002a). By using improved test-particle codes (see Qin et al. 2002b for instance), it has been demonstrated that for a non-slab model, diffusion is recovered (though only partially, as demonstrated in Sect. 3 of this paper). This recovery of diffusion cannot been explained by the method of Kóta \& Jokipii (2000).

Various other theories have been proposed for perpendicular transport, mainly based on nonlinear extensions of QLT, such as the nonlinear closure approximation of Owens (1974) or model-based approaches such as the one proposed by Bieber \& Matthaeus (BAM 1997). However, these theories provide a diffusive behavior of perpendicular transport for the slab model, in disagreement with simulations. One more promising theory, namely the nonlinear guiding-center theory (NLGCtheory), has been derived by Matthaeus et al. (2003). Although this theory shows agreement with some test-particle simulations in slab/2D geometries, the theory cannot reproduce subdiffusion for the slab model. An extended nonlinear guiding-center (ENLGC) theory was therefore formulated by Shalchi (2006), which agrees with simulations for slab and non-slab models. However, this theory is very close to the original NLGC-theory and uses nearly the same crude approximation: exponential form of the velocity correlation function, magnetic fields and particle velocities are uncorrelated.

In this paper we propose a more reliable theoretical approach that uses less ad-hoc assumptions and ansätze than the NLGCtheory. The layout of this article goes as follows. In Sect. 2, we argue that field-line wandering behaves superdiffusively for non-slab models. In Sect. 3, we employ a generalized compound diffusion model to deduce an analytic form for the perpendicular MSD of particles. By comparing with test-particle simulations (Sect. 4) and solar wind observations (Sect. 5) we show that our theory provides the correct result.

\section{Nonlinear description of field-line wandering}

The key input into our new formulation is the MSD of the magnetic field-lines $\left\langle(\Delta x(z))^{2}\right\rangle_{\mathrm{FL}}$. In several previous papers (e.g. 
Jokipii 1966; Matthaeus et al. 1995; Ragot 2006; Ruffolo et al. 2006), field-line wandering (or random walk) has been described by applying linear and nonlinear formulations. In a recent paper (Shalchi \& Kourakis 2007), an improved formulation for nonlinear field-line random walk in magnetostatic turbulence has been developed, thus criticizing the validity of those previous approaches. The approach of Shalchi \& Kourakis (2007) is a direct generalization of the theory proposed by Matthaeus et al. (1995) and further applied by Ruffolo et al. (2006). For a diffusive behavior of field-lines this theory can be obtained by the theory of Shalchi \& Kourakis (2007) as a special limit. However, our theory can also be applied in non-diffusive transport cases. A further advantage of this theory is its analytical tractability, which enriches and complements the existing numerical toolbox on field-line wandering (see e.g. Ragot 2006).

In view of modeling field-line random walk, the turbulence model has to be specified in terms of the magnetic correlation tensor $P_{i j}(\boldsymbol{k})=\left\langle\delta B_{i}(\boldsymbol{k}) \delta B_{j}(\boldsymbol{k})\right\rangle$. According to Bieber et al. (1994) the so-called slab/2D composite model is a realistic model for solar wind turbulence. This two-component model ignores the usually smaller parallel field turbulent variance $\left(\delta B_{z}\right)$ and only includes excitations with wavevectors either purely parallel or purely perpendicular to the mean magnetic field $\boldsymbol{B}_{0}$, leading to the following form of the correlation tensors: $P_{x x}(\boldsymbol{k})=P_{x x}^{\text {slab }}(\boldsymbol{k})+P_{x x}^{2 \mathrm{D}}(\boldsymbol{k})$ with $P_{x x}^{\text {slab }}(\boldsymbol{k})=g^{\text {slab }}\left(k_{\|}\right) \delta\left(k_{\perp}\right) / k_{\perp}$ and $P_{x x}^{2 \mathrm{D}}(\boldsymbol{k})=g^{2 \mathrm{D}}\left(k_{\perp}\right) \delta\left(k_{\|}\right) k_{y}^{2} / k_{\perp}^{3}$. For the two wave spectra $g^{\text {slab }}\left(k_{\|}\right)$ and $g^{2 \mathrm{D}}\left(k_{\perp}\right)$, we employ a standard form that has also been proposed by Bieber et al. (1994)

$g^{\text {slab }}\left(k_{\|}\right)=\frac{C(v)}{2 \pi} l_{\text {slab }} \delta B_{\text {slab }}^{2}\left(1+k_{\|}^{2} l_{\text {slab }}^{2}\right)^{-v}$

$g^{2 \mathrm{D}}\left(k_{\perp}\right)=\frac{2 C(v)}{\pi} l_{2 \mathrm{D}} \delta B_{2 \mathrm{D}}^{2}\left(1+k_{\perp}^{2} l_{2 \mathrm{D}}^{2}\right)^{-v}$.

Here we have defined the normalization constant $C(v)=$ $\Gamma(v) /(2 \sqrt{\pi} \Gamma(v-1 / 2))$, where $\Gamma$ denotes the Euler Gamma function. We have defined the slab- and $2 \mathrm{D}$ bendover length scales $l_{\text {slab }}$ and $l_{2 \mathrm{D}}$, the strength of the turbulent fields $\delta B_{\text {slab }}$ and $\delta B_{2 \mathrm{D}}$, and the inertial-range spectral index $2 v$.

It can easily be demonstrated (see e.g. Jokipii 1966; Matthaeus et al. 1995) that, for pure slab geometry, the fieldlines behaves diffusively

$\left\langle(\Delta x(z))^{2}\right\rangle_{|z| \rightarrow \infty} \approx 2 \kappa_{\mathrm{FL}}|z|$,

for large $|z|$. In several previous papers (e.g. Matthaeus et al. 1995) it has been explicitly assumed that the form of Eq. (2) also holds in two-component turbulence. More precisely, field-line wandering always behaves diffusively, at least as long as there is a finite slab contribution. However, by applying an improved formulation of field-line random walk (Shalchi \& Kourakis 2007), it has been shown that

$$
\begin{aligned}
\left\langle(\Delta x(z))^{2}\right\rangle_{|z| \rightarrow \infty}= & \left(9 \sqrt{\frac{\pi}{2}} C(v)\right)^{2 / 3} \\
& \times\left(\frac{\delta B_{2 \mathrm{D}}}{B_{0}}\right)^{4 / 3} l_{2 \mathrm{D}}^{2}\left(\frac{|z|}{l_{2 \mathrm{D}}}\right)^{4 / 3} .
\end{aligned}
$$

The only assumptions that have been applied to derive this result are Corrsin's independence hypothesis (Corrsin 1959) and the assumption of a Gaussian distribution of field-lines (see Matthaeus et al. 1995). For two-component turbulence, Eq. (3) is the correct asymptotic limit that can be obtained in the limit $z \rightarrow \infty$. A quasilinear description of the field-line random walk is not possible for these length scales (see Shalchi \& Kourakis 2007). For completeness, it should be noted that the different regimes where a quasilinear description is valid or not are discussed in Isichenko (1991a,b).

\section{Generalized compound diffusion of charged particles}

In the previous section, we discussed results regarding the fieldline wandering in the slab/2D composite model as a function of $z$. However, charged particles experience parallel scattering while moving through the turbulence. Thus, the parameter $z$ becomes a statistical variable in particle transport studies. If we assume that the particles (or, more precisely, their guiding-centers) follow the magnetic field-lines (guiding center approximation), we have

$\left\langle(\Delta x(t))^{2}\right\rangle_{P}=\int_{-\infty}^{+\infty} \mathrm{d} z\left\langle(\Delta x(z))^{2}\right\rangle_{\mathrm{FL}} f_{P}(z, t)$.

Here the index $P$ denotes the perpendicular MSD of the charged particle, and $f_{P}(z, t)$ is the particle distribution in the direction parallel to the background field.

Equation (4) can also be obtained from the ChapmanKolmogorov equation (see, e.g., Webb et al. 2006), which has the form

$f_{\perp}(x, t)=\int_{-\infty}^{+\infty} \mathrm{d} z f_{\mathrm{FL}}(x, z) f_{P}(z, t)$

with the particle distribution in the perpendicular direction $f_{\perp}(x, t)$ and the field-line distribution function $f_{\mathrm{FL}}(x, z)$. By calculating the second moment of $f_{\perp}(x, t)$, Eq. (4) can be deduced from Eq. (5).

A further standard assumption in the cosmic ray transport theory is the assumption of a Gaussian particle distribution, see e.g. Matthaeus et al. (2003):

$f_{P}(z, t)=\left(2 \pi\left\langle(\Delta z(t))^{2}\right\rangle_{P}\right)^{-1 / 2} \mathrm{e}^{-\frac{z^{2}}{2\left\langle(\Delta z(t))^{2}\right\rangle_{P}}}$.

It should be noted, however, that the assumption of a Gaussian distribution is a certain hypothesis that might be inaccurate for certain parameter regimes. A non-Gaussian distribution might be more appropriate mainly for a strong non-diffusivity of parallel particle propagation. However, as demonstrated in Sect. 4, the real particle motion is very close to the diffusive behavior, thus Eq. (6) should be a good approximation. Forthcoming work will be devoted to exploring the influence of non-Gaussian statistics.

By using Eq. (3) for the field-line MSD in combination with Eq. (6) for the particle distribution, we can evaluate Eq. (4) to find

$\left\langle(\Delta x)^{2}\right\rangle_{P}=\alpha(v)\left(\frac{\delta B_{2 \mathrm{D}}}{B_{0}}\right)^{4 / 3}\left[l_{2 \mathrm{D}}\left\langle(\Delta z(t))^{2}\right\rangle_{P}\right]^{2 / 3}$,

with

$\alpha(v)=\frac{\Gamma(7 / 6)}{\sqrt{\pi}}\left(18 \sqrt{\frac{\pi}{2}} C(v)\right)^{2 / 3}$

This is a tractable analytical result that can easily be applied to the parameter regimes considered in test particle simulations or for turbulence parameters appropriate for the solar wind. In observed spectra, it was clearly found that $v=5 / 6$ and thus $\alpha(v=5 / 6) \approx 0.5$. A (time-dependent) diffusion coefficient as obtained from test-particle simulations can be defined 
as $\kappa_{x x}(t)=\left\langle(\Delta x)^{2}\right\rangle /(2 t)$. In general, one may adopt the assumption $\left\langle(\Delta z(t))^{2}\right\rangle_{P} \sim t^{b_{\|}+1}$, implying a parallel diffusion coefficient $\kappa_{z z} \sim t^{b_{\|}}$. By assuming $\kappa_{x x} \sim t^{b_{\perp}}$, it is straightforward to find from Eq. (7) the relation

$b_{\perp}=\frac{2 b_{\|}-1}{3}$.

Therefore, knowledge of $b_{\|}$(e.g., from simulation data) leads to an evaluation of $b_{\perp}$, within this model. For instance, if parallel transport behaves diffusively $\left(b_{\|}=0\right)$, we find $b_{\perp}=-1 / 3$ (subdiffusion). A diffusive behavior of perpendicular transport $\left(b_{\perp}=0\right)$ can only be obtained for $b_{\|}=1 / 2$ (superdiffusion).

For pure slab geometry, however, we deduce by combining Eqs. (2) and (6) with Eq. (4):

$\left.\left\langle(\Delta x)^{2}\right\rangle_{P}\right|_{t \rightarrow \infty}=2 \sqrt{\frac{2}{\pi}} \kappa_{\mathrm{FL}} \sqrt{\left\langle(\Delta z(t))^{2}\right\rangle_{P}}$

and therefore

$b_{\perp}^{\text {slab }}=\frac{b_{\|}^{\text {slab }}-1}{2}$.

For parallel diffusion $\left(b_{\|}^{\text {slab }}=0\right.$ ), we cleary obtain the wellknown result $b_{\perp}^{\text {slab }}=-1 / 2$ (see e.g. Kóta \& Jokipii 2000; Shalchi 2005). While the slab result is well-known, the relations (7) and (9) are entirely new. The formulation proposed by this paper allows a systematic and reliable discription of perpendicular transport requiring knowledge of parallel transport. We refer to this new approach as the Generalized Compound Diffusion $(G C D)$-model. In the next section, the GCD-model will be tested via test-particle simulations.

\section{Test particle simulations}

In this section numerical tests are employed to assess the accuracy of the GCD-model. By choosing the same turbulence model as used for the application of the GCD-model (magnetostatic turbulence, slab/2D composite geometry, the wave spectra of Eq. (1)) test-particle simulations can be performed easily. The diffusion coefficient is computed numerically using procedures described previously (Giacalone \& Jokipii 1999; Qin et al. 2002a,b).

We have considered test-particle dynamics for the following set of parameters: $l_{2 \mathrm{D}}=0.1 l_{\mathrm{slab}}, v=5 / 6$, and $20 \% / 80 \%$ slab/2D composite geometry. In Fig. 1, we depict the ratio of perpendicular and parallel diffusion coefficients $\kappa_{x x} / \kappa_{z z}$ as a function of the dimensionless time $\tau=v t / l_{\text {slab }}$ for the dimensionless cosmic ray rigidity value $R=R_{L} / l_{\text {slab }}=0.001$. We have chosen a low value of $R$ to ensure that the guiding-center approximation is valid. The new results are compared to those obtained from the NLGC- and ENLGC-theories and also to test-particle simulations. For the NLGC-results we have assumed a parameter value of $a^{2}=1$, which corresponds to the assumption that guidingcenters follow magnetic field-lines. Obviously the GCD-model provides a result much closer to the simulations than the NLGCtheory and the ENLGC-theory. However, it should be emphasized that the main advantage of the GCD-model is its more systematic nature.

By assuming the simple form $\tilde{\kappa}_{x x}(t)=a \tau^{b}$, we can deduce the time dependence from numerical data by using $b=\left(\ln \tilde{\kappa}_{x x}(\tau)-\right.$ $\ln a) / \ln \tau \approx\left(\ln \tilde{\kappa}_{x x}(\tau)\right) / \ln \tau$ in the high time limit $\left(\tilde{\kappa}_{x x}\right.$ denotes the dimensionless diffusion coefficients obtained by the simulations). The exponents for the parallel $b_{\|}$and perpendicular $b_{\perp}$

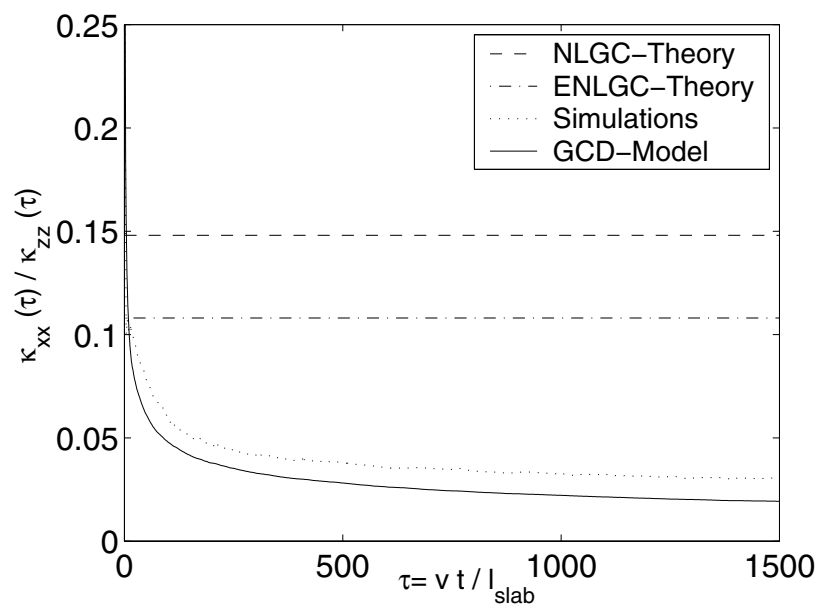

Fig. 1. The ratio of perpendicular and parallel diffusion coefficients $\left(\kappa_{x x}(t) / \kappa_{z z}(t)\right)$ for $R=R_{L} / l_{\text {slab }}=0.001$. The results from test-particle simulations (dotted line) are compared to various theoretical results: NLGC-theory (dashed line), ENLGC-theory (dash-dotted line), and our GCD-model (solid line).

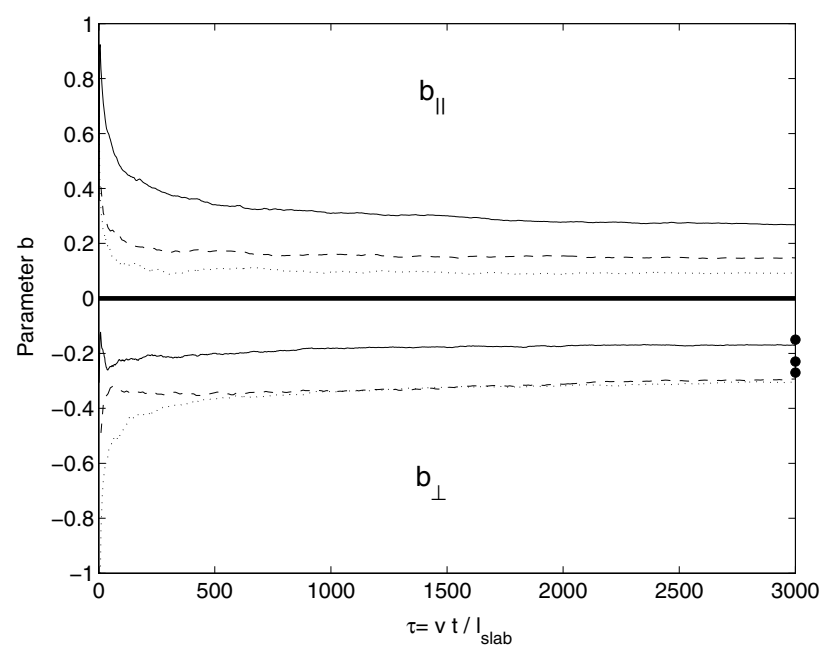

Fig. 2. The parameters $b_{\|}$and $b_{\perp}$ as a function of time for different values of the dimensionless rigidity: $R=10^{-3}$ (dotted line), $R=10^{-2}$ (dashed line), and $R=10^{-1}$ (solid line). The dots denote the values predicted by the GCD-model. Clearly we find a weakly superdiffusive behavior of parallel transport $\left(b_{\|}>0\right)$ and a weakly subdiffusive behavior of perpendicular transport $\left(b_{\perp}<0\right)$.

diffusion coefficients are depicted in Fig. 2 for different values of the parameter $R$. As shown, the test particle code provides a weakly superdiffusive behavior of parallel transport, in addition to a weakly subdiffusive behavior of perpendicular transport. In all cases considered, the GCD-model agrees well with the simulations.

\section{Comparison with solar wind observations}

It is difficult to directly compare our new (non-diffusive) result with solar wind observations. In this section, we attempt a rough comparison by averaging our non-diffusive result over the characteristic scattering time

$t_{\mathrm{c}}=\frac{\lambda_{\|}}{v}$

where we have defined the parallel mean free path $\lambda_{\|}$and the velocity $v$ of the charged particle. First, we replace the 
parallel mean-square deviation in Eq. (7) by a diffusive behavior $\left(\left\langle(\Delta z(t))^{2}\right\rangle_{P} \approx 2 t \kappa_{\|}\right)$to get

$\left\langle(\Delta x)^{2}\right\rangle_{P}=\alpha(v)\left(\frac{\delta B_{2 \mathrm{D}}}{B_{0}}\right)^{4 / 3}\left(2 l_{2 \mathrm{D}} t \kappa_{\|}\right)^{2 / 3}$

and thus one obtains for the (time-dependent) perpendicular diffusion coefficient

$\kappa_{\perp}(t)=2^{-1 / 3} \alpha(v)\left(\frac{\delta B_{2 \mathrm{D}}}{B_{0}}\right)^{4 / 3}\left(l_{2 \mathrm{D}} \kappa_{\|}\right)^{2 / 3} t^{-1 / 3}$.

To proceed, we average over the scattering time (see Eq. (12)) to get

$$
\begin{aligned}
\bar{\kappa}_{\perp} & =\frac{1}{t_{\mathrm{c}}} \int_{0}^{t_{\mathrm{c}}} \kappa_{\perp}(t) \\
& =\frac{3}{2^{4 / 3}} \alpha(v)\left(\frac{\delta B_{2 \mathrm{D}}}{B_{0}}\right)^{4 / 3}\left(l_{2 \mathrm{D}} \kappa_{\|}\right)^{2 / 3}\left(\frac{v}{\lambda_{\|}}\right)^{1 / 3} .
\end{aligned}
$$

By using $\lambda_{\|}=3 \kappa_{\|} / v$ and $\lambda_{\perp}=3 \kappa_{\perp} / v$, we find an analytical expression for the perpendicular mean free path

$\bar{\lambda}_{\perp}=\left(\frac{3}{2}\right)^{4 / 3} \alpha(v)\left(\frac{\delta B_{2 \mathrm{D}}}{B_{0}}\right)^{4 / 3} l_{2 \mathrm{D}}^{2 / 3} \lambda_{\|}^{1 / 3}$

For $v=5 / 6$ and $\delta B_{2 \mathrm{D}}^{2} / B_{0}^{2}=0.8$, as proposed by Bieber et al. (1994), we obtain

$\bar{\lambda}_{\perp}=0.75 l_{2 \mathrm{D}}^{2 / 3} \lambda_{\|}^{1 / 3}$.

Palmer (1982) suggested that the parallel mean free path in the solar wind is $0.08 \mathrm{AU} \leq \lambda_{\|, \text {Palmer }} \leq 0.3 \mathrm{AU}$ and the perpendicular spatial diffusion coefficient is $\kappa_{\perp} c / v \approx 10^{21} \mathrm{~cm}^{2} \mathrm{~s}^{-1}$ and thus $\lambda_{\perp, \text { Palmer }} \approx 0.007 \mathrm{AU}$. By taking the average value for the parallel mean free path $\lambda_{\|, \text {Palmer }} \approx 0.2$ and by applying Eq. (17) we find $\lambda_{\perp, \mathrm{GCD}} \approx 0.009 \mathrm{AU}$ (for $l_{2 \mathrm{D}}=0.1 l_{\text {slab }} \approx 0.003 \mathrm{AU}$, as suggested by e.g. Matthaeus et al. 2003), which is therefore very close to the measurements. Obviously there is good agreement between solar wind observations and our new theoretical approach.

The values for the perpendicular mean free paths obtained from Jovian electrons (Chenette et al. 1977) and Ulysses measurements of Galactic protons (Burger et al. 2000) are similar. Thus we conclude that the generalized compound diffusion model can reproduce solar wind observations for the perpendicular mean free paths.

To reproduce these observations we applied Eq. (16), which can be obtained from the more general result of Eq. (7) by averaging over the scattering time. According to our new theory and test-particle simulations, parallel as well as perpendicular transport behaves non-diffusively. However, the nondiffusivity is very weak, thus Eqs. (16) and (17) should be good approximations.

\section{Summary and conclusion}

By combining a compound diffusion model - cf. Eq. (4) - with a nonlinear treatment of field-line wandering (namely Eq. (3)), a new theoretical treatment for the perpendicular transport of cosmic rays is presented in this article. In Table 1, the assumptions of this new theory are compared to the NLGC-theory, as representative of existing transport theories. Obviously the new approach relies on less approximations and model assumptions. Therefore the GCD-model is less restricted and thus more reliable. In Table 2, we compare different theories and their results
Table 1. Comparison between the assumptions used in our GCD-model and the assumptions used in the NLGC-theory.

\begin{tabular}{lll}
\hline \hline Assumption & NLGC & GCD \\
\hline Guiding-center approximation & YES & YES \\
Gaussian statistics & YES & YES \\
Corrsin's hypothesis & YES & YES \\
Velocities and fields are uncorrelated & YES & NO \\
Exponential velocity correlation & YES & NO \\
Diffusion approximation & YES & NO \\
\hline
\end{tabular}

Table 2. Comparison between results for the parameter $b_{\perp}$ from various theories: QLT, BAM-model, NLGC-theory, WNLT, ENLGC-theory, and the GCD-model. Negative values of $b_{\perp}$ correspond to subdiffusion, positive values to superdiffusion, and $b_{\perp}=0$ corresponds to diffusion.

\begin{tabular}{lll}
\hline \hline Theory & slab turbulence & slab/2D composite \\
\hline Simulations & -0.5 & $\approx-0.2$ \\
\hline QLT & 0 & 1.0 \\
BAM & 0 & 0 \\
NLGC & 0 & 0 \\
WNLT & 0 & 0 \\
ENLGC & $-1 / 2$ & 0 \\
GCD-model & $-1 / 2$ & $\approx-0.2$ \\
\hline
\end{tabular}

for the parameter $b_{\perp}$, which denotes the time dependence of the diffusion coefficient via $\kappa_{x x}(t) \sim t^{b_{\perp}}$. Furthermore, the theory is easily applicable due to its simple analytical form (see Eqs. (7) and (8)).

Through comparison with direct numerical simulations of test particles, we have demonstrated that the GCD-model behaves very well and provides a noticeably improved description of perpendicular transport compared to several other theories considered in the tables for reference. Furthermore, by averaging over the scattering time, we have derived a simple formula (Eq. (16)) for the perpendicular mean free path. This formula can easily be applied for solar wind parameters and can be compared with observations. As demonstrated, there is very good agreement between the GCD-model and the observations discussed by Chenette et al. (1977), Palmer (1982), and Burger et al. (2000), similar to the results of the NLGC-theory and its extended version. However, the NLGC-theory, as well as the ENLGC-theory, predict very large perpendicular mean free paths for certain limits (see Bieber et al. 2004; Shalchi 2006). These limits do not exist as shown in the current article, hence Eqs. (7) and (8) represent perpendicular tranport for all parameter regimes within the two-component model. Thus, besides the weak superdiffusivity discovery in the article, the GCD-model clearly disagrees with the NLGC-theory that has been applied in several transport theory studies. It is the subject of our current work to study the application of the present theory in space physics and astrophysics in the hope that an improved formulation of perpendicular transport might be useful in solving a number of observational puzzles. Mainly the non-diffusivity of particle transport for turbulence models that have been considered in the past as realistic models for solar wind turbulence could be important for reproducing heliospheric observations.

Acknowledgements. This research was supported by the Deutsche Forschungsgemeinschaft (DFG) under the Emmy-Noether Programm (grant SH 93/3-1). As a member of the Junges Kolleg, A. Shalchi also aknowledges support by the Nordrhein-Westfälische Akademie der Wissenschaften. The authors are grateful to Prof. W. H. Matthaeus, 
Dr. G. Qin, and especially Dr. J. Minnie of the Bartol Research Institute and Department of Physics and Astronomy, University of Delaware for providing the test-particle code used in this paper. Futher information of the code can be found at http://www.bartol.udel.edu/\%7Ewhmgroup/ Streamline/streamline.html

\section{References}

Bieber, J. W., \& Matthaeus, W. H. 1997, ApJ, 485, 655

Bieber, J. W., Matthaeus, W. H., Smith, C. W., et al. 1994, ApJ, 420, 294

Bieber, J. W., Matthaeus, W. H., Shalchi, A., \& Qin, G. 2004, Geophys. Res. Lett., 31, L10805

Burger, R. A., \& Hattingh, M. 1998, ApJ, 505, 244

Burger, R. A., Potgieter, M. S., \& Heber, B. 2000, J. Geophys. Res., 105, 27447 Chenette, D. L., Conlon, T. F., Pyle, K. R., \& Simpson, J. A. 1977, ApJ, 215, L95

Corrsin, S. 1959, in Atmospheric Diffusion and Air Pollution, Advanced in Geophysics, Vol. 6, ed. F. Frenkiel, \& P. Sheppard (New York: Academic)

Dröge, W. 2000, Space Sci. Rev., 93, 121

Giacalone, J., \& Jokipii, J. R. 1999, ApJ, 520, 204

Isichenko, M. B. 1991a, Plasma Physics and Controlled Fusion, 33, 795
Isichenko, M. B. 1991b, Plasma Physics and Controlled Fusion, 33, 809 Jokipii, J. R. 1966, ApJ, 146, 480

Jokipii, J. R., \& Parker, E. N. 1969, ApJ, 155, 799

Kóta, J., \& Jokipii, J. R. 2000, ApJ, 531, 1067

Kubo, R. 1957, J. Phys. Soc. Japan, 12, 570

Mason, G. M., Desai, M. I., Cohen, C. M. S., et al. 2006, ApJ, 647, L65

Matthaeus, W. H., Gray, P. C., Pontius, D. H. Jr., \& Bieber, J. W. 1995, Phys. Rev. Lett., 75, 2136

Matthaeus, W. H., Qin, G., Bieber, J. W., \& Zank, G. P. 2003, ApJ, 590, L53

Owens, A. J. 1974, ApJ, 191, 235

Palmer, I. D. 1982, Rev. Geophys. Space Phys., 20, 335

Qin, G., Matthaeus, W. H., \& Bieber, J. W. 2002a, Geophys. Res. Lett., 29

Qin, G., Matthaeus, W. H., \& Bieber, J. W. 2002b, ApJ, 578, L117

Ragot, B. R. 2006, ApJ, 644, 622

Ruffolo, D., Chuychai, P., \& Matthaeus, W. H. 2006, ApJ, 644, 971

Shalchi, A. 2005, J. Geophys. Res., 110, A09103

Shalchi, A. 2006, A\&A, 453, L43

Shalchi, A., \& Kourakis, I. 2007, Phys. Plasmas, submitted [arXiv: astro-ph/0703366]

Shalchi, A., Bieber, J. W., Matthaeus, W. H., \& Qin, G. 2004, ApJ, 616, 617

Webb, G. M., Zank, G. P., Kaghashvili, E. Kh., \& leRoux, J. A. 2006, ApJ, 651, 211 